\title{
Raoultella ornithinolytica
}

National Cancer Institute

\section{Source}

National Cancer Institute. Raoultella ornithinolytica. NCI Thesaurus. Code C86715.

A species of Gram negative, rod shaped bacteria assigned to the phylum Proteobacteria.

This species is ornithine decarboxylase positive, able to grow at 10 degrees Celsius and uses sorbose as a carbon source. R. ornithinolytica is a pathogen that can cause enteric fever like syndrome and histamine fish poisoning. 